Supporting Information for

\title{
Zinc and Cadmium Complexation of L-Threonine: An Infrared Multiple Photon Dissociation Spectroscopy and Theoretical Study
}

Georgia C. Boles,,$^{\dagger}$ Randy L. Hightower, ${ }^{\dagger}$ Giel Berden, ${ }^{+}$Jos Oomens, ${ }^{+, \#}$ and P. B. Armentrout ${ }^{*, \dagger}$

${ }^{\dagger}$ Department of Chemistry, University of Utah, 315 S. 1400 E. Rm. 2020, Salt Lake City, Utah 84112, United States

Radboud University, Institute for Molecules and Materials, FELIX Laboratory, Toernooiveld 7 , NL-6525 ED Nijmegen, The Netherlands

\#van't Hoff Institute for Molecular Sciences, University of Amsterdam, Science Park 904, NL1098 XH Amsterdam, The Netherlands

* Corresponding author, e-mail: armentrout@chem.utah.edu

Tables S1 and S2 provide scaled vibrational frequencies for the five lowest-lying conformers of $\mathrm{Zn}(\mathrm{Thr}-\mathrm{H})^{+}(\mathrm{ACN})$ and key geometric parameters for the located $\mathrm{Zn}(\mathrm{Thr}-\mathrm{H})^{+}(\mathrm{ACN})$ structures, respectively. Tables S3 and S4 provide relative energies and key geometric parameters for located $\mathrm{Zn}(\mathrm{Thr}-\mathrm{H})^{+}$structures, respectively. Table S5 gives key geometric parameters for the located $\mathrm{Zn}(\mathrm{Gly}-\mathrm{H})^{+}(\mathrm{ACN})$ species. Tables S6 - S8 provide relative energies for higher-lying $\mathrm{CdCl}^{+}(\mathrm{Thr})$ structures, scaled vibrational frequencies for the five lowest-lying $\mathrm{CdCl}^{+}(\mathrm{Thr})$ structures, and the key geometric parameters for the located $\mathrm{CdCl}^{+}(\mathrm{Thr})$ species, respectively. Figure $\mathrm{S} 1$ gives structures of higher energy $\mathrm{Zn}(\mathrm{Thr}-\mathrm{H})^{+}(\mathrm{ACN})$ complexes and its isomers. Figure S2 compares the experimental $\mathrm{Zn}(\mathrm{Thr}-\mathrm{H})^{+}(\mathrm{ACN})$ spectrum with the calculated spectra for $\left[\mathrm{N}, \mathrm{CO}^{-}\right]-\operatorname{gtt}(\mathrm{ACN}),\left[\mathrm{N}^{-}, \mathrm{CO}, \mathrm{OH}_{\mathrm{s}}\right]-\operatorname{tggt}(\mathrm{ACN})$, and the sum of $\left[\mathrm{N}, \mathrm{CO}^{-}, \mathrm{OH}_{\mathrm{s}}\right]-\operatorname{ggt}(\mathrm{ACN})$ and $\left[\mathrm{N}, \mathrm{O}_{\mathrm{s}}^{-}\right.$ $, \mathrm{OH}]-\operatorname{tgg}(\mathrm{ACN})$. Figures S3 - S5 show structures of higher energy $\mathrm{Zn}(\mathrm{Thr}-\mathrm{H})^{+}$, $\mathrm{Zn}(\mathrm{Gly}-\mathrm{H})^{+}(\mathrm{ACN})$, and $\mathrm{CdCl}^{+}(\mathrm{Thr})$ complexes, respectively. 


\section{Figure Captions}

Figure S1. Structures of select higher-energy $\mathrm{Zn}(\mathrm{Thr}-\mathrm{H})^{+}(\mathrm{ACN})$ conformers calculated at the B3LYP/6-311+G(d,p) level of theory. Short dashed lines indicate hydrogen bonds. Metal-ligand interactions are shown by long dashed lines. (Red—oxygen, grey—carbon, white-hydrogen, blue—nitrogen, steel grey—zinc.)

Figure S2. Comparison of the $\mathrm{Zn}(\mathrm{Thr}-\mathrm{H})^{+}(\mathrm{ACN})$ experimental IRMPD action spectrum with the calculated IR spectra for $\left[\mathrm{N}, \mathrm{CO}^{-}\right]-\operatorname{gtt}(\mathrm{ACN}),\left[\mathrm{N}^{-}, \mathrm{CO}, \mathrm{OH}_{\mathrm{s}}\right]-\operatorname{tggt}(\mathrm{ACN})$, and the sum of $\left[\mathrm{N}, \mathrm{CO}^{-}\right.$ , $\left.\mathrm{OH}_{\mathrm{s}}\right]-\operatorname{ggt}(\mathrm{ACN})$ and $\left[\mathrm{N}, \mathrm{O}_{\mathrm{s}}^{-}, \mathrm{OH}\right]-\operatorname{tgg}(\mathrm{ACN})$ calculated at the $\mathrm{B} 3 \mathrm{LYP} / 6-311+\mathrm{G}(\mathrm{d}, \mathrm{p})$ level of theory. Relative $298 \mathrm{~K}$ Gibbs energies in $\mathrm{kJ} / \mathrm{mol}$ are given at the B3LYP, B3P86, and MP2(full) levels, respectively.

Figure S3. Structures of select higher-energy $\mathrm{Zn}(\mathrm{Thr}-\mathrm{H})^{+}$conformers calculated at the B3LYP/6-311+G(d,p) level of theory. Short dashed lines indicate hydrogen bonds. Metal-ligand interactions are shown by long dashed lines. (Red—oxygen, grey—carbon, white-hydrogen, blue—nitrogen, steel grey—zinc.)

Figure S4. Structures of select higher-energy $\mathrm{Zn}(\mathrm{Gly}-\mathrm{H})^{+}(\mathrm{ACN})$ conformers calculated at the B3LYP/6-311+G(d,p) level of theory. Short dashed lines indicate hydrogen bonds. Metal-ligand interactions are shown by long dashed lines. (Red-oxygen, grey-carbon, white-hydrogen, blue—nitrogen, steel grey—zinc.)

Figure S5. Structures of select $\mathrm{CdCl}^{+}(\mathrm{Thr})$ conformers calculated at the B3LYP/def2-TZVP level of theory. Short dashed lines indicate hydrogen bonds. Metal-ligand interactions are shown by long dashed lines. (Red —oxygen, grey—carbon, white—hydrogen, blue—nitrogen, yellow— cadmium, green - chlorine.) 
Table S1. Vibrational frequencies $\left(\mathrm{cm}^{-1}\right)$ scaled by 0.975 and IR intensities $(\mathrm{km} / \mathrm{mol}$, in parentheses) for the five lowest energy $\mathrm{Zn}(\mathrm{Thr}-\mathrm{H})^{+}(\mathrm{ACN})$ conformers calculated at B3LYP/6$311+\mathrm{G}(\mathrm{d}, \mathrm{p})$ level of theory. ${ }^{a}$

\begin{tabular}{|c|c|c|c|c|c|c|c|c|c|c|c|}
\hline \multicolumn{4}{|c|}{$\left[\mathrm{N}, \mathrm{O}_{\mathrm{s}}^{-}, \mathrm{CO}\right]-\operatorname{tgg}(\mathrm{ACN})$} & \multicolumn{2}{|c|}{$\begin{array}{c}\left.\mathrm{N}, \mathrm{CO}^{-}, \mathrm{OH}_{\mathrm{s}}\right]- \\
\operatorname{ggt}(\mathrm{ACN}) \\
\end{array}$} & \multicolumn{2}{|c|}{$\begin{array}{c}{\left[\mathrm{N}, \mathrm{O}_{\mathrm{s}}^{-}, \mathrm{CO}\right]-} \\
\operatorname{cgg}(\mathrm{ACN}) \\
\end{array}$} & \multicolumn{2}{|c|}{$\begin{array}{c}{\left[\mathrm{N}, \mathrm{O}_{\mathrm{s}}^{-}\right]-} \\
\operatorname{tg} \mathrm{t}(\mathrm{ACN}) \\
\end{array}$} & \multicolumn{2}{|c|}{$\begin{array}{l}{\left[\mathrm{N}, \mathrm{CO}^{-}\right]-} \\
\operatorname{gtt}(\mathrm{ACN})\end{array}$} \\
\hline 24 & $(<1)$ & 24 & $(<1)$ & 13 & $(<1)$ & 17 & $(<1)$ & 24 & $(<1)$ & 23 & $(<1)$ \\
\hline 32 & (4) & 32 & (4) & 31 & (3) & 32 & (5) & 27 & (1) & 26 & (1) \\
\hline 37 & (4) & 37 & (5) & 38 & (1) & 36 & (7) & 33 & (3) & 34 & (2) \\
\hline 78 & (4) & 78 & (4) & 78 & (5) & 78 & (3) & 46 & (2) & 52 & (1) \\
\hline 120 & (1) & 120 & (1) & 106 & (3) & 119 & (1) & 89 & (9) & 70 & (8) \\
\hline 139 & (3) & 139 & (3) & 137 & (1) & 141 & (4) & 99 & $(<1)$ & 107 & $(<1)$ \\
\hline 147 & (8) & 146 & (8) & 146 & (7) & 146 & (7) & 144 & (7) & 148 & (11) \\
\hline 161 & (20) & 159 & (20) & 169 & (36) & 168 & (33) & 158 & (12) & 159 & (10) \\
\hline 199 & (6) & 199 & (6) & 200 & (4) & 205 & (18) & 184 & (16) & 190 & (13) \\
\hline 220 & (5) & 220 & (5) & 219 & (5) & 218 & (3) & 196 & (6) & 217 & (10) \\
\hline 232 & (1) & 232 & (1) & 231 & (4) & 232 & (2) & 221 & (1) & 224 & (122) \\
\hline 262 & (6) & 261 & (6) & 263 & (62) & 257 & (6) & 267 & (10) & 242 & (1) \\
\hline 305 & (21) & 303 & (22) & 292 & (38) & 302 & (14) & 289 & (7) & 299 & (12) \\
\hline 331 & (24) & 329 & (22) & 308 & (16) & 330 & (24) & 325 & (12) & 316 & (17) \\
\hline 353 & (20) & 352 & (18) & 327 & (30) & 354 & (10) & 365 & (16) & 355 & (33) \\
\hline 383 & (2) & 383 & (2) & 369 & (14) & 383 & (14) & 410 & (2) & 380 & $(<1)$ \\
\hline 407 & (2) & 407 & (2) & 408 & (2) & 407 & (2) & 410 & (2) & 410 & (2) \\
\hline 409 & (2) & 409 & (2) & 410 & (2) & 409 & (2) & 442 & (7) & 411 & (3) \\
\hline 467 & (18) & 467 & (18) & 438 & (7) & 466 & (8) & 495 & (11) & 427 & (24) \\
\hline 566 & (17) & 564 & (17) & 444 & (15) & 513 & (108) & 517 & $(21)$ & 466 & (2) \\
\hline 587 & (18) & 587 & (18) & 493 & (18) & 572 & (32) & 593 & (72) & 504 & (13) \\
\hline 606 & (61) & 606 & (61) & 616 & (13) & 587 & (6) & 621 & (23) & 574 & (6) \\
\hline 647 & (110) & 647 & (109) & 660 & (28) & 640 & (2) & 647 & (72) & 650 & (31) \\
\hline 692 & (35) & 692 & (35) & 698 & (15) & 673 & (46) & 658 & $(48)$ & 691 & (9) \\
\hline 704 & (28) & 704 & (28) & 752 & (30) & 713 & (13) & 710 & (33) & 761 & (66) \\
\hline 807 & (25) & 807 & (25) & 805 & (28) & 801 & (7) & 839 & (8) & 852 & (6) \\
\hline
\end{tabular}




\begin{tabular}{|c|c|c|c|c|c|c|c|c|c|c|c|}
\hline \multicolumn{4}{|c|}{$\left[\mathrm{N}, \mathrm{O}_{\mathrm{s}}{ }^{-}, \mathrm{CO}\right]-\operatorname{tgg}(\mathrm{ACN})$} & \multicolumn{2}{|c|}{$\begin{array}{c}{\left[\mathrm{N}, \mathrm{CO}^{-}, \mathrm{OH}_{\mathrm{s}}\right]-} \\
\operatorname{ggt}(\mathrm{ACN})\end{array}$} & \multicolumn{2}{|c|}{$\begin{array}{c}{\left[\mathrm{N}, \mathrm{O}_{\mathrm{s}}^{-}, \mathrm{CO}\right]-} \\
\operatorname{cgg}(\mathrm{ACN})\end{array}$} & \multicolumn{2}{|c|}{$\begin{array}{c}{\left[\mathrm{N}, \mathrm{O}_{\mathrm{s}}^{-}\right]^{-}} \\
\operatorname{tg} \mathrm{t}(\mathrm{ACN}) \\
\end{array}$} & \multicolumn{2}{|c|}{$\begin{array}{l}{\left[\mathrm{N}, \mathrm{CO}^{-}\right]-} \\
\mathrm{gtt}(\mathrm{ACN})\end{array}$} \\
\hline 870 & (12) & 870 & (12) & 855 & (24) & 871 & (25) & 884 & (85) & 873 & (5) \\
\hline 892 & (41) & 892 & (41) & 888 & (18) & 892 & (29) & 900 & (39) & 900 & (28) \\
\hline 936 & (8) & 936 & (8) & 936 & (11) & 937 & (8) & 938 & (10) & 939 & (11) \\
\hline 972 & (43) & 972 & (43) & 946 & (23) & 976 & (54) & 991 & (130) & 978 & (44) \\
\hline 1009 & (134) & 1009 & (134) & 997 & (18) & 1002 & (109) & 1022 & (8) & 1021 & (24) \\
\hline 1023 & (7) & 1023 & (7) & 1022 & (7) & 1023 & (7) & 1023 & (7) & 1021 & (7) \\
\hline 1025 & (7) & 1025 & (7) & 1024 & (7) & 1025 & (7) & 1033 & (10) & 1022 & (8) \\
\hline 1049 & (52) & 1049 & (52) & 1051 & (115) & 1049 & (26) & 1081 & (11) & 1063 & (5) \\
\hline 1071 & (5) & 1071 & (5) & 1062 & (59) & 1069 & (21) & 1101 & (158) & 1089 & (75) \\
\hline 1092 & (5) & 1092 & (5) & 1098 & (12) & 1091 & (9) & 1136 & (7) & 1132 & (305) \\
\hline 1161 & (132) & 1161 & (132) & 1136 & (36) & 1157 & (10) & 1159 & (238) & 1185 & (71) \\
\hline 1181 & (114) & 1181 & (114) & 1205 & (128) & 1178 & (120) & 1182 & (33) & 1217 & (199) \\
\hline 1246 & (16) & 1246 & (16) & 1238 & (164) & 1239 & (14) & 1224 & (4) & 1227 & (14) \\
\hline 1266 & (7) & 1266 & (7) & 1265 & (21) & 1267 & (194) & 1319 & (53) & 1276 & (21) \\
\hline 1319 & (11) & 1319 & (11) & 1316 & (5) & 1315 & (46) & 1329 & (20) & 1324 & (12) \\
\hline 1343 & (19) & 1343 & (19) & 1350 & (4) & 1337 & (56) & 1341 & (25) & 1337 & (6) \\
\hline 1368 & (9) & 1368 & (9) & 1352 & (13) & 1368 & (9) & 1366 & (10) & 1365 & (11) \\
\hline 1371 & (5) & 1371 & (5) & 1367 & (10) & 1372 & (11) & 1380 & (18) & 1377 & (3) \\
\hline 1414 & (18) & 1414 & (18) & 1389 & (14) & 1387 & (198) & 1398 & (19) & 1387 & (18) \\
\hline 1414 & (18) & 1414 & (18) & 1412 & (18) & 1414 & (18) & 1411 & (18) & 1409 & (19) \\
\hline 1423 & (117) & 1423 & (117) & 1412 & (18) & 1414 & (18) & 1411 & (19) & 1409 & (19) \\
\hline 1438 & (10) & 1438 & (10) & 1450 & (7) & 1437 & (4) & 1448 & (8) & 1456 & (10) \\
\hline 1461 & (9) & 1461 & (9) & 1461 & (8) & 1460 & (8) & 1459 & (3) & 1462 & (6) \\
\hline 1603 & (93) & 1603 & (93) & 1601 & (64) & 1603 & (75) & 1610 & (47) & 1597 & (54) \\
\hline 1663 & (385) & 1663 & (385) & 1752 & (507) & 1687 & (302) & 1751 & (301) & 1755 & (430) \\
\hline 2339 & (202) & 2339 & (202) & 2336 & $(225)$ & 2340 & (208) & 2333 & (232) & 2332 & (263) \\
\hline 2906 & (30) & 2906 & (30) & 2962 & (6) & 2879 & (44) & 2883 & (35) & 2902 & (34) \\
\hline 2947 & (19) & 2947 & (19) & 2968 & (21) & 2947 & (17) & 2967 & $(25)$ & 2962 & (11) \\
\hline
\end{tabular}




\begin{tabular}{|c|c|c|c|c|c|c|c|c|c|c|c|}
\hline \multicolumn{4}{|c|}{$\left[\mathrm{N}, \mathrm{O}_{\mathrm{s}}^{-}, \mathrm{CO}\right]-\operatorname{tgg}(\mathrm{ACN})$} & \multicolumn{2}{|c|}{$\begin{array}{c}{\left[\mathrm{N}, \mathrm{CO}^{-}, \mathrm{OH}_{\mathrm{s}}\right]-} \\
\operatorname{ggt}(\mathrm{ACN})\end{array}$} & \multicolumn{2}{|c|}{$\begin{array}{c}{\left[\mathrm{N}, \mathrm{O}_{\mathrm{s}}^{-}, \mathrm{CO}\right]-} \\
\operatorname{cgg}(\mathrm{ACN}) \\
\end{array}$} & \multicolumn{2}{|c|}{$\begin{array}{c}{\left[\mathrm{N}, \mathrm{O}_{\mathrm{s}}^{-}\right]^{-}} \\
\operatorname{tg} \mathrm{t}(\mathrm{ACN}) \\
\end{array}$} & \multicolumn{2}{|c|}{$\begin{array}{l}{\left[\mathrm{N}, \mathrm{CO}^{-}\right]-} \\
\operatorname{gtt}(\mathrm{ACN})\end{array}$} \\
\hline 2969 & (18) & 2969 & (18) & 3007 & $(<1)$ & 2969 & (18) & 2967 & $(8)$ & 2966 & (27) \\
\hline 3010 & (28) & 3010 & (28) & 3029 & (2) & 2986 & (12) & 3015 & (3) & 3021 & (1) \\
\hline 3029 & (7) & 3029 & (7) & 3032 & (15) & 3009 & (36) & 3038 & (15) & 3028 & (14) \\
\hline 3043 & (5) & 3043 & (5) & 3038 & (5) & 3043 & (5) & 3043 & (10) & 3043 & (11) \\
\hline 3045 & (8) & 3045 & (8) & 3044 & (9) & 3045 & (8) & 3044 & (9) & 3043 & (10) \\
\hline 3046 & (7) & 3046 & (7) & 3045 & (8) & 3046 & (7) & 3052 & (6) & 3053 & (2) \\
\hline 3403 & (21) & 3403 & (21) & 3393 & $(20)$ & 3403 & $(21)$ & 3309 & (73) & 3309 & (120) \\
\hline 3470 & (38) & 3470 & (38) & 3458 & (37) & 3472 & (38) & 3432 & (43) & 3422 & (56) \\
\hline 3629 & (161) & 3629 & (161) & 3740 & (124) & 3686 & (116) & 3638 & (142) & 3741 & (79) \\
\hline
\end{tabular}

${ }^{a}$ Bold indicates the region of the IR spectrum included in the present experimental study. Values in italics were calculated using ${ }^{68} \mathrm{Zn}$ in order to evaluate possible band broadening as a result of different $\mathrm{Zn}$ isotopes monitored for the yield calculation. 
Table S2. Bond Distances $(\AA)$ and Bond Angles (deg) for $\mathrm{Zn}(\mathrm{Thr}-\mathrm{H})^{+}(\mathrm{ACN})$ Structures $^{a}$

\begin{tabular}{|c|c|c|c|c|c|c|c|}
\hline structure & $\mathrm{r}(\mathrm{M}-\mathrm{X})$ & $\mathrm{r}(\mathrm{M}-\mathrm{O})$ & $\mathrm{r}\left(\mathrm{M}-\mathrm{O}_{\mathrm{s}}\right)$ & $\mathrm{r}(\mathrm{M}-\mathrm{N})$ & $\angle \mathrm{XMO}$ & $\angle \mathrm{XMO}_{\mathrm{s}}$ & $\angle \mathrm{OMO}_{\mathrm{s}}$ \\
\hline$\left[\mathrm{N}, \mathrm{O}_{\mathrm{s}}^{-}, \mathrm{CO}\right]-\operatorname{tgg}(\mathrm{ACN})$ & 2.16 & 2.19 & 1.87 & 1.96 & 76.1 & 85.7 & 94.8 \\
\hline$\left[\mathrm{N}, \mathrm{CO}^{-}, \mathrm{OH}_{\mathrm{s}}\right]-\operatorname{ggt}(\mathrm{ACN})$ & 2.09 & 1.90 & 2.14 & 1.96 & 85.3 & 78.1 & 95.0 \\
\hline$\left[\mathrm{N}, \mathrm{O}_{\mathrm{s}}^{-}, \mathrm{CO}\right]-\operatorname{cgg}(\mathrm{ACN})$ & 2.18 & 2.16 & 1.89 & 1.96 & 76.1 & 84.7 & 96.1 \\
\hline$\left[\mathrm{N}, \mathrm{O}_{\mathrm{s}}^{-}\right]-\operatorname{tg} \_\mathrm{t}(\mathrm{ACN})$ & 2.07 & & 1.82 & 1.95 & & 90.1 & \\
\hline$\left[\mathrm{N}, \mathrm{CO}^{-}\right]-\operatorname{gtt}(\mathrm{ACN})$ & 2.03 & 1.86 & & 1.94 & 90.0 & & \\
\hline$\left[\mathrm{N}, \mathrm{O}_{\mathrm{s}}^{-}, \mathrm{OH}\right]-\operatorname{tgg}(\mathrm{ACN})$ & 2.12 & $2.39^{b}$ & 1.84 & 1.96 & $70.4^{b}$ & 88.7 & $90.9^{b}$ \\
\hline$\left[\mathrm{N}, \mathrm{CO}^{-}\right]-\operatorname{ttt}(\mathrm{ACN})$ & 2.03 & 1.86 & & 1.94 & 89.4 & & \\
\hline$\left[\mathrm{N}, \mathrm{O}_{\mathrm{s}}^{-}\right]-\operatorname{tg}_{+} \mathrm{t}(\mathrm{ACN})$ & 2.09 & & 1.82 & 1.95 & & 89.9 & \\
\hline$\left[\mathrm{CO}^{-}, \mathrm{O}_{\mathrm{s}}^{-}\right]\left(\mathrm{NH}_{3}{ }^{+}\right)-\operatorname{cg}(\mathrm{ACN})$ & & 1.95 & 1.86 & 1.95 & & & 100.7 \\
\hline$\left[\mathrm{N}^{-}, \mathrm{CO}, \mathrm{OH}_{\mathrm{s}}\right]-\operatorname{tggt}(\mathrm{ACN})$ & 1.88 & 2.18 & 2.30 & 1.95 & 82.3 & 83.3 & 79.9 \\
\hline$\left[\mathrm{N}, \mathrm{CO}^{-}\right]-\operatorname{tgg}(\mathrm{ACN})$ & 2.04 & 1.86 & & 1.94 & 89.6 & & \\
\hline$\left[\mathrm{N}, \mathrm{O}_{\mathrm{s}}^{-}\right]-\operatorname{cgt}(\mathrm{ACN})$ & 2.07 & & 1.83 & 1.95 & & 89.9 & \\
\hline$\left[\mathrm{N}, \mathrm{O}_{\mathrm{s}}^{-}\right]-\operatorname{ccg}(\mathrm{ACN})$ & 2.10 & & 1.85 & 1.95 & & 90.3 & \\
\hline$\left[\mathrm{CO}_{2}^{-}\right]-\operatorname{ggt}(\mathrm{ACN})$ & $1.99^{c}$ & 1.97 & & 1.92 & $67.0^{c}$ & & \\
\hline$\left[\mathrm{N}, \mathrm{C}_{\gamma}^{-}\right]-\operatorname{tg} \_\mathrm{g} \_\mathrm{t}(\mathrm{ACN})$ & 2.16 & & $1.97^{d}$ & 2.00 & & $90.6^{d}$ & \\
\hline$\left[\mathrm{CO}^{-}, \mathrm{OH}_{\mathrm{s}}\right]-\operatorname{cgc}(\mathrm{ACN})$ & & 1.84 & 2.01 & 1.94 & & & 100.8 \\
\hline$\left[\mathrm{N}^{-}, \mathrm{CO}\right]-\operatorname{tgtt}(\mathrm{ACN})$ & 1.82 & 2.10 & & 1.93 & 84.9 & & \\
\hline$\left[\mathrm{N}, \mathrm{C}_{\gamma}^{-}\right]-\mathrm{tg}_{+} \mathrm{g}_{+} \mathrm{t}(\mathrm{ACN})$ & 2.17 & & $1.97^{d}$ & 2.00 & & $90.8^{d}$ & \\
\hline$\left[\mathrm{CO}_{2}^{-}\right]-\operatorname{gtt}(\mathrm{ACN})$ & $1.98^{c}$ & 1.98 & & 1.92 & $67.0^{c}$ & & \\
\hline$\left[\mathrm{CO}_{2}{ }^{-}, \mathrm{OH}_{\mathrm{s}}\right]-\mathrm{gcg}(\mathrm{ACN})$ & $2.07^{c}$ & 2.00 & 2.19 & 1.94 & $65.6^{c}$ & $82.6^{c}$ & 83.9 \\
\hline$\left[\mathrm{N}^{-}, \mathrm{OH}_{\mathrm{s}}\right]-\operatorname{tttt}(\mathrm{ACN})$ & 1.83 & & 2.07 & 1.94 & & 85.3 & \\
\hline$\left[\mathrm{N}^{-}, \mathrm{OH}_{\mathrm{s}}\right]-\operatorname{tgtt}(\mathrm{ACN})$ & 1.81 & & 2.09 & 1.93 & & 84.5 & \\
\hline$\left[\mathrm{N}^{-}, \mathrm{OH}_{\mathrm{s}}, \mathrm{OH}\right]-\operatorname{tggt}(\mathrm{ACN})$ & 1.86 & $2.31^{b}$ & 2.20 & 1.95 & $77.4^{b}$ & 86.0 & $78.7^{b}$ \\
\hline$\left[\mathrm{O}_{\mathrm{s}}^{-}, \mathrm{OH}\right]-\operatorname{tgg}(\mathrm{ACN})$ & & $2.11^{b}$ & 1.79 & 1.94 & & & 98.4 \\
\hline $\begin{array}{l}{\left[\mathrm{N}^{-}, \mathrm{OH}\right]-\operatorname{tgtt}(\mathrm{ACN})} \\
\quad \text { Values calculate } \\
\text { amino nitrogen, } \mathrm{O}=\text { carb } \\
\text { nitrogen of } \mathrm{ACN} \text { ligand. } \\
\text { carboxylate group. }{ }^{d} \mathrm{O}_{\mathrm{s}}=\end{array}$ & $\begin{array}{l}1.81 \\
\text { at the B } \\
\text { yl oxyg } \\
\partial=\text { oxy }\end{array}$ & $\begin{array}{l}2.16^{b} \\
\text { LYP/6-3 }\end{array}$ & $\begin{array}{l}1+\mathrm{G}(\mathrm{d}, \mathrm{p} \\
\text { one, } \mathrm{O}_{\mathrm{s}}\end{array}$ & $\begin{aligned} & 1.92 \\
& \text { level o }= \\
&= \text { side-cl } \\
& \text { drovyl }\end{aligned}$ & $\begin{array}{l}82.6^{b} \\
\text { theory. } \\
\text { in hydr }\end{array}$ & $\begin{array}{l}\text { xcept as } \\
\text { xyl oxyge }\end{array}$ & 1oted, $\mathrm{X}$ \\
\hline
\end{tabular}


Table S3. Relative Energies (0 K) and Gibbs Energies (298 K) of Zn(Thr-H) ${ }^{+}$Complexes $^{a}$

\begin{tabular}{|c|c|c|c|}
\hline structure & B3LYP/GD3BJ ${ }^{b}$ & B3P86 & MP2(full) \\
\hline$\left[\mathrm{N}, \mathrm{O}_{\mathrm{s}}^{-}, \mathrm{CO}\right] \operatorname{tgg}$ & $0.0(0.0) / \mathbf{0 . 0}(\mathbf{0 . 0})$ & $0.0(0.0)$ & $0.0(0.0)$ \\
\hline$\left[\mathrm{N}, \mathrm{CO}^{-}, \mathrm{OH}_{\mathrm{s}}\right] \mathrm{ggt}$ & $12.5(11.8) / \mathbf{1 0 . 8}(\mathbf{9 . 9})$ & $15.4(14.6)$ & $9.5(8.8)$ \\
\hline$\left[\mathrm{N}, \mathrm{O}_{\mathrm{s}}^{-}, \mathrm{CO}\right] \mathrm{cgg}$ & $21.6(21.5) / 21.1(\mathbf{2 1 . 0})$ & $21.0(21.0)$ & $21.7(21.7)$ \\
\hline$\left[\mathrm{N}, \mathrm{C}_{\gamma}^{-}\right] \mathrm{tg} \_\mathrm{g} \_\mathrm{t}$ & $36.4(32.7) / 36.4(\mathbf{3 2 . 7})$ & $40.3(36.5)$ & $38.9(35.2)$ \\
\hline$\left[\mathrm{O}_{\mathrm{s}}^{-}, \mathrm{CO}\right] \mathrm{cgg}$ & $43.2(41.7)$ & $45.9(44.4)$ & $53.1(51.6)$ \\
\hline$\left[\mathrm{N}, \mathrm{C}_{\gamma}^{-}\right] \mathrm{tg}_{+} \mathrm{g}_{+} \mathrm{t}$ & $44.7(40.2)$ & $49.9(45.5)$ & $48.9(44.4)$ \\
\hline$\left[\mathrm{N}, \mathrm{O}_{\mathrm{s}}^{-}, \mathrm{OH}\right] \operatorname{tgg}$ & $46.1(45.1)$ & $50.4(49.4)$ & $42.2(41.2)$ \\
\hline$\left[\mathrm{N}, \mathrm{O}_{\mathrm{s}}^{-}\right] \operatorname{tg} \_\mathrm{t}$ & $50.3(46.0)$ & $58.2(53.9)$ & $58.9(54.6)$ \\
\hline$\left[\mathrm{N}^{-}, \mathrm{CO}, \mathrm{OH}_{\mathrm{s}}\right] \operatorname{tggt}$ & $51.4(50.3)$ & $54.3(53.2)$ & $54.3(53.1)$ \\
\hline$\left[\mathrm{CO}^{-}, \mathrm{O}^{-}\right]\left(\mathrm{NH}_{3}^{+}\right) \mathrm{ctg}$ & $51.7(49.9)$ & $57.7(55.9)$ & $55.3(53.4)$ \\
\hline$\left[\mathrm{N}, \mathrm{O}_{\mathrm{s}}^{-}\right] \operatorname{tg}_{+} \mathrm{t}$ & $62.9(58.6)$ & $72.5(68.2)$ & $70.9(66.6)$ \\
\hline$\left[\mathrm{N}, \mathrm{CO}^{-}\right]-\mathrm{gtt}$ & $67.3(63.0)$ & $75.0(70.6)$ & $74.1(69.7)$ \\
\hline$\left[\mathrm{O}_{\mathrm{s}}^{-}, \mathrm{CO}\right] \operatorname{tg}_{+} \mathrm{g}_{+}$ & $72.1(69.5)$ & $79.5(76.8)$ & $84.4(81.8)$ \\
\hline$\left[\mathrm{O}_{\mathrm{s}}^{-}, \mathrm{CO}\right] \operatorname{tg} \_\mathrm{g}$ & $73.0(70.1)$ & $81.1(78.1)$ & $87.0(84.1)$ \\
\hline$\left[\mathrm{N}^{-}, \mathrm{CO}\right]-\operatorname{tgtt}$ & $89.4(84.6)$ & $98.3(93.5)$ & $104.7(99.9)$ \\
\hline$\left[\mathrm{N}, \mathrm{CO}^{-}\right]-\operatorname{tgg}$ & $95.5(92.1)$ & $103.2(99.8)$ & $104.5(101.2)$ \\
\hline$\left[\mathrm{N}^{-}, \mathrm{OH}_{\mathrm{s}}, \mathrm{OH}\right]-\operatorname{tggt}$ & $95.6(93.6)$ & $103.0(101.0)$ & $96.6(94.6)$ \\
\hline$\left[\mathrm{O}^{-}, \mathrm{O}_{\mathrm{s}}\right] \mathrm{ggt}$ & $103.1(98.6)$ & $114.8(110.4)$ & $114.1(109.7)$ \\
\hline$\left[\mathrm{N}^{-}, \mathrm{O}_{\mathrm{s}}\right]-\mathrm{tttt}$ & $103.6(98.9)$ & $116.7(112.0)$ & $115.7(111.0)$ \\
\hline$\left[\mathrm{CO}_{2}^{-}\right]$-ggt & $104.7(97.6)$ & $122.6(115.4)$ & $160.0(152.8)$ \\
\hline$\left[\mathrm{N}, \mathrm{CO}^{-}\right]$-ggt & $107.5(103.5)$ & $112.6(108.6)$ & $112.1(108.1)$ \\
\hline$\left[\mathrm{CO}_{2}{ }^{-}, \mathrm{OH}_{\mathrm{s}}\right]-\mathrm{gcg}$ & $109.6(108.5)$ & $114.5(113.4)$ & $121.6(120.5)$ \\
\hline$\left[\mathrm{N}^{-}, \mathrm{O}_{\mathrm{s}}\right]-\mathrm{tctt}$ & $113.2(106.5)$ & $126.3(119.6)$ & $122.3(115.6)$ \\
\hline$\left[\mathrm{CO}_{2}^{-}\right]-$gtg & $116.0(109.1)$ & $134.0(127.1)$ & $177.4(170.5)$ \\
\hline
\end{tabular}

${ }^{a}$ Relative single point energies at $0 \mathrm{~K}$ and $298 \mathrm{~K}$ Gibbs energies (in parentheses) calculated at the level of theory indicated using a $6-311+\mathrm{G}(2 \mathrm{~d}, 2 \mathrm{p})$ basis set. ${ }^{b}$ Empirical dispersion corrected B3LYP-GD3BJ values are given in bold. 
Table S4. Bond Distances $(\AA)$ and Bond Angles (deg) for $\mathrm{Zn}(\mathrm{Thr}-\mathrm{H})^{+}$Structures $^{a}$

\begin{tabular}{|c|c|c|c|c|c|c|}
\hline structure & $\mathrm{r}(\mathrm{M}-\mathrm{X})$ & $\mathrm{r}(\mathrm{M}-\mathrm{O})$ & $\mathrm{r}\left(\mathrm{M}-\mathrm{O}_{\mathrm{s}}\right)$ & $\angle \mathrm{XMO}$ & $\angle \mathrm{XMO}_{\mathrm{s}}$ & $\angle \mathrm{OMO}_{\mathrm{s}}$ \\
\hline$\left[\mathrm{N}, \mathrm{O}_{\mathrm{s}}^{-}, \mathrm{CO}\right] \operatorname{tgg}$ & 2.12 & 2.10 & 1.86 & 79.3 & 89.8 & 100.2 \\
\hline$\left[\mathrm{N}, \mathrm{CO}^{-}, \mathrm{OH}_{s}\right] \mathrm{ggt}$ & 2.06 & 1.89 & 2.06 & 89.2 & 81.1 & 101.1 \\
\hline$\left[\mathrm{N}, \mathrm{O}_{\mathrm{s}}^{-}, \mathrm{CO}\right] \operatorname{cgg}$ & 2.13 & 2.07 & 1.86 & 79.2 & 88.7 & 101.9 \\
\hline$\left[\mathrm{N}, \mathrm{C}_{\gamma}{ }^{-}\right] \mathrm{tg} \_\mathrm{g} \_\mathrm{t}$ & 2.09 & & $2.00^{b}$ & & $96.0^{b}$ & \\
\hline$\left[\mathrm{O}_{\mathrm{s}}^{-}, \mathrm{CO}\right] \mathrm{cgg}$ & & 1.93 & 1.82 & & & 112.3 \\
\hline$\left[\mathrm{N}, \mathrm{C}_{\gamma}^{-}\right] \operatorname{tg}_{+} \mathrm{g}_{+} \mathrm{t}$ & 2.11 & & $2.00^{b}$ & & $96.0^{b}$ & \\
\hline$\left[\mathrm{N}, \mathrm{O}_{\mathrm{s}}^{-}, \mathrm{OH}\right] \operatorname{tgg}$ & 2.08 & $2.21^{c}$ & 1.85 & $74.9^{c}$ & 93.5 & $96.7^{c}$ \\
\hline$\left[\mathrm{N}, \mathrm{O}_{\mathrm{s}}^{-}\right] \operatorname{tg} \_\mathrm{t}$ & 2.02 & & 1.82 & & 96.4 & \\
\hline$\left[\mathrm{N}^{-}, \mathrm{CO}, \mathrm{OH}_{\mathrm{s}}\right] \operatorname{tggt}$ & 1.91 & 2.10 & 2.17 & 85.1 & 88.4 & 84.2 \\
\hline$\left[\mathrm{CO}^{-}, \mathrm{O}^{-}\right]\left(\mathrm{NH}_{3}^{+}\right) \mathrm{ctg}$ & & 1.88 & 1.84 & & & 110.2 \\
\hline$\left[\mathrm{N}, \mathrm{O}_{\mathrm{s}}^{-}\right] \operatorname{tg}_{+} \mathrm{t}$ & 2.04 & & 1.82 & & & 95.8 \\
\hline$\left[\mathrm{N}, \mathrm{CO}^{-}\right]$-gtt & 1.99 & 1.85 & & 95.9 & & \\
\hline$\left[\mathrm{O}_{\mathrm{s}}^{-}, \mathrm{CO}\right] \mathrm{tg}_{+} \mathrm{g}_{+}$ & & 1.95 & 1.81 & & & 111.1 \\
\hline$\left[\mathrm{O}_{\mathrm{s}}^{-}, \mathrm{CO}\right] \operatorname{tg} \_\mathrm{g}$ & & 1.95 & 1.81 & & & 111.0 \\
\hline$\left[\mathrm{N}^{-}, \mathrm{CO}\right]-\operatorname{tgtt}$ & 1.87 & 2.05 & & 88.5 & & \\
\hline$\left[\mathrm{N}, \mathrm{CO}^{-}\right]-\operatorname{tgg}$ & 2.01 & 1.85 & & 95.2 & & \\
\hline$\left[\mathrm{N}^{-}, \mathrm{OH}_{\mathrm{s}}, \mathrm{OH}\right]$-tggt & 1.90 & $2.16^{c}$ & 2.12 & $81.1^{c}$ & 90.6 & $82.9^{c}$ \\
\hline$\left[\mathrm{O}^{-}, \mathrm{O}_{\mathrm{s}}\right] \mathrm{ggt}$ & & 1.82 & 1.92 & & & 112.7 \\
\hline$\left[\mathrm{N}^{-}, \mathrm{O}_{\mathrm{s}}\right]-\mathrm{tttt}$ & 1.87 & & 2.01 & & 89.3 & \\
\hline$\left[\mathrm{CO}_{2}^{-}\right]$-ggt & $2.04^{d}$ & 2.03 & & $65.3^{d}$ & & \\
\hline$\left[\mathrm{N}, \mathrm{CO}^{-}\right]$-ggt & 2.03 & 1.85 & & 93.5 & & \\
\hline$\left[\mathrm{CO}_{2}{ }^{-}, \mathrm{OH}_{\mathrm{s}}\right]-\mathrm{gcg}$ & $2.05^{d}$ & 2.00 & 2.10 & $66.2^{d}$ & $84.9^{d}$ & 85.8 \\
\hline$\left[\mathrm{N}^{-}, \mathrm{O}_{\mathrm{s}}\right]$-tctt & 1.87 & & 2.01 & & 89.3 & \\
\hline$\left[\mathrm{CO}_{2}^{-}\right]-\mathrm{gtg}$ & $2.06^{d}$ & 2.04 & & $65.0^{d}$ & & \\
\hline
\end{tabular}

${ }^{a}$ Values calculated at the B3LYP/6-311+G(d,p) level of theory. Except as noted, $\mathrm{X}=$ amino nitrogen, $\mathrm{O}=$ carbonyl oxygen of backbone, and $\mathrm{O}_{\mathrm{s}}=$ side-chain hydroxyl oxygen. ${ }^{b} \mathrm{O}_{\mathrm{s}}=$ $\mathrm{C}_{\gamma}{ }^{-} \cdot{ }^{c} \mathrm{O}=$ oxygen of backbone hydroxyl group. ${ }^{d} \mathrm{X}=$ second oxygen of carboxylate group. 
Table S5. Bond Distances $(\AA)$ and Bond Angles (deg) for $\mathrm{Zn}(\mathrm{Gly}-\mathrm{H})^{+}(\mathrm{ACN})$ Structures $^{a}$

\begin{tabular}{|c|c|c|c|c|}
\hline structure & $\mathrm{r}(\mathrm{M}-\mathrm{X})$ & $\mathrm{r}(\mathrm{M}-\mathrm{O})$ & $\mathrm{r}(\mathrm{M}-\mathrm{N})$ & $\angle \mathrm{XMO}$ \\
\hline $\mathrm{Zn}(\mathrm{Gly}-\mathrm{H})^{+}\left[\mathrm{N}, \mathrm{CO}^{-}\right]-\mathrm{c}(\mathrm{ACN})$ & 2.06 & 1.85 & 1.94 & 90.4 \\
\hline $\mathrm{Zn}(\mathrm{Gly}-\mathrm{H})^{+}\left[\mathrm{N}^{-}, \mathrm{CO}\right]-\mathrm{tt}(\mathrm{ACN})$ & 1.83 & 2.10 & 1.93 & 85.5 \\
\hline $\mathrm{Zn}(\mathrm{Gly}-\mathrm{H})^{+}\left[\mathrm{CO}_{2}^{-}\right]-\mathrm{c}(\mathrm{ACN})$ & $1.99^{b}$ & 1.98 & 1.92 & $66.8^{b}$ \\
\hline $\mathrm{Zn}(\mathrm{Gly}-\mathrm{H})^{+}\left[\mathrm{N}^{-}, \mathrm{CO}\right]-\operatorname{ct}(\mathrm{ACN})$ & 1.83 & 2.08 & 1.93 & 85.7 \\
\hline $\mathrm{Zn}(\mathrm{Gly}-\mathrm{H})^{+}\left[\mathrm{CO}_{2}^{-}\right]-\mathrm{c}, \mathrm{NH} \cdot \mathrm{CO}(\mathrm{ACN})$ & 1.98 & 1.98 & 1.92 & 66.9 \\
\hline $\mathrm{Zn}(\mathrm{Gly}-\mathrm{H})^{+}[\mathrm{N}, \mathrm{CO}]\left(\mathrm{C}_{\alpha^{-}}^{-}\right)-\mathrm{tt}(\mathrm{ACN})$ & 2.04 & 1.87 & 1.94 & 90.4 \\
\hline $\mathrm{Zn}(\mathrm{Gly}-\mathrm{H})^{+}[\mathrm{N}, \mathrm{CO}]\left(\mathrm{C}_{\alpha^{-}}^{-}\right)-\operatorname{ct}(\mathrm{ACN})$ & 2.05 & 1.86 & 1.94 & 90.1 \\
\hline $\mathrm{Zn}(\mathrm{Gly}-\mathrm{H})^{+}\left[\mathrm{N}^{-}, \mathrm{OH}\right]-\operatorname{tc}(\mathrm{ACN})$ & 1.81 & $2.14^{c}$ & 1.92 & $83.2^{c}$ \\
\hline $\mathrm{Zn}(\mathrm{Gly}-\mathrm{H})^{+}\left[\mathrm{C}_{\alpha}^{-}, \mathrm{CO}\right]-\mathrm{cc}(\mathrm{ACN})$ & $2.03^{d}$ & 2.23 & 1.97 & $67.0^{d}$ \\
\hline $\mathrm{Zn}(\mathrm{Gly}-\mathrm{H})^{+}\left[\mathrm{C}_{\alpha}^{-}, \mathrm{CO}\right]-\mathrm{tc}(\mathrm{ACN})$ & $2.01^{d}$ & 2.35 & 1.97 & $65.4^{d}$ \\
\hline
\end{tabular}

${ }^{a}$ Values calculated at the B3LYP/6-311+G(d,p) level of theory. Except as noted, $\mathrm{X}=$ amino nitrogen, $\mathrm{O}=$ carbonyl oxygen of backbone, and $\mathrm{N}=$ nitrogen of $\mathrm{ACN} .{ }^{b} \mathrm{X}=$ second oxygen of carboxylate group. ${ }^{c} \mathrm{O}=$ oxygen of backbone hydroxyl group. ${ }^{d} \mathrm{X}=\mathrm{C}_{\alpha}$. 
Table S6. Relative Energies (0 K) and Gibbs Energies (298 K) of $\mathrm{CdCl}^{+}(\mathrm{Thr}) \mathrm{Complexes}^{a}$

\begin{tabular}{|c|c|c|c|}
\hline structure & B3LYP & В3Р86 & MP2(full) \\
\hline$\left[\mathrm{CO}^{-}, \mathrm{OH}_{\mathrm{s}}\right]-\mathrm{ggt}$ & $60.5(59.1)$ & $61.4(60.1)$ & $70.8(69.4)$ \\
\hline$\left[\mathrm{N}, \mathrm{OH}_{\mathrm{s}}\right]$-cgtt & $62.0(60.8)$ & $64.3(63.0)$ & $68.8(67.5)$ \\
\hline$\left[\mathrm{CO}, \mathrm{OH}_{\mathrm{s}}\right]-\mathrm{tg}_{+} \mathrm{g}_{+} \mathrm{t}$ & $62.8(59.6)$ & $68.2(65.1)$ & $78.9(75.7)$ \\
\hline$\left[\mathrm{CO}, \mathrm{OH}_{\mathrm{s}}\right]-\operatorname{tg} \mathrm{g}_{-} \mathrm{t}$ & $65.4(62.4)$ & $71.3(68.3)$ & $83.3(80.3)$ \\
\hline$\left[\mathrm{O}_{\mathrm{s}}^{-}\right]-\operatorname{tgg}$ & $65.6(62.8)$ & $68.7(65.9)$ & $79.1(76.3)$ \\
\hline$[\mathrm{CO}]-\mathrm{ccg}_{+} \mathrm{t}$ & $67.5(65.1)$ & $72.6(70.2)$ & $94.6(92.1)$ \\
\hline$[\mathrm{CO}]-\operatorname{ccg}_{-} \mathrm{t}$ & $70.3(67.6)$ & $73.9(71.2)$ & $93.2(90.6)$ \\
\hline$[\mathrm{CO}]$-cggt & $73.2(67.8)$ & $76.4(71.0)$ & $95.5(90.0)$ \\
\hline$\left[\mathrm{O}_{\mathrm{s}}^{-}\right]-\mathrm{ttg}$ & 80.7 (77.6) & $85.2(82.1)$ & $93.4(90.3)$ \\
\hline$[\mathrm{N}, \mathrm{OH}] \operatorname{tggg}$ & $92.8(89.3)$ & $95.9(92.4)$ & $93.9(90.4)$ \\
\hline$[\mathrm{N}, \mathrm{OH}] \operatorname{tggt}$ & $93.1(90.1)$ & $96.6(93.6)$ & $94.0(91.0)$ \\
\hline$\left[\mathrm{O}_{\mathrm{s}}^{-}, \mathrm{OH}\right]-\operatorname{tgg}$ & $105.0(101.1)$ & $108.5(104.5)$ & $118.8(114.8)$ \\
\hline$\left[\mathrm{OH}_{\mathrm{s}}, \mathrm{OH}\right] \operatorname{tggt}$ & $108.5(102.5)$ & $117.9(111.9)$ & $122.1(116.1)$ \\
\hline$\left[\mathrm{O}_{\mathrm{s}}^{-}, \mathrm{CO}\right]-\operatorname{tgg}$ & $111.8(108.6)$ & $116.4(113.2)$ & $125.0(121.8)$ \\
\hline
\end{tabular}


Table S7. Vibrational frequencies $\left(\mathrm{cm}^{-1}\right)$ scaled by 0.975 and IR intensities $(\mathrm{km} / \mathrm{mol}$, in parentheses) for the five lowest energy conformers of $\mathrm{CdCl}^{+}(\mathrm{Thr})$ calculated at B3LYP/def2TZVP level of theory ${ }^{a}$

\begin{tabular}{|c|c|c|c|c|c|c|c|c|c|}
\hline \multicolumn{2}{|c|}{$\left[\mathrm{N}, \mathrm{CO}, \mathrm{OH}_{\mathrm{s}}\right]-\operatorname{tggt}$} & \multicolumn{2}{|c|}{$[\mathrm{N}, \mathrm{CO}]-\mathrm{tg} \mathrm{g}_{+} \mathrm{t}$} & \multicolumn{2}{|c|}{$\left[\mathrm{CO}_{2}{ }^{-}\right]-\mathrm{ctgt}$} & \multicolumn{2}{|c|}{$\left[\mathrm{CO}_{2}^{-}\right]-\mathrm{cgtt}$} & \multicolumn{2}{|c|}{$[\mathrm{N}, \mathrm{CO}]$-tgtt } \\
\hline 42 & (2) & 32 & (2) & 37 & $(<1)$ & 38 & $(<1)$ & 28 & (3) \\
\hline 47 & (3) & 45 & (4) & 46 & (2) & 44 & $(<1)$ & 46 & (5) \\
\hline 67 & (3) & 63 & (1) & 60 & (8) & 52 & (1) & 51 & (1) \\
\hline 101 & (1) & 80 & (1) & 68 & (2) & 63 & (10) & 69 & $(<1)$ \\
\hline 125 & (6) & 110 & (3) & 92 & (2) & 89 & (8) & 106 & (3) \\
\hline 143 & (4) & 144 & (2) & 108 & (9) & 99 & (2) & 157 & (7) \\
\hline 189 & (1) & 180 & (8) & 157 & (14) & 169 & (16) & 186 & (2) \\
\hline 213 & (2) & 205 & (3) & 227 & (5) & 230 & (2) & 211 & (3) \\
\hline 235 & (1) & 232 & (1) & 242 & $(10)$ & 234 & (13) & 238 & $(10)$ \\
\hline 243 & (106) & 282 & (7) & 266 & (15) & 255 & (24) & 242 & (112) \\
\hline 291 & (12) & 312 & (99) & 296 & (95) & 281 & (104) & 304 & (9) \\
\hline 322 & (13) & 329 & (11) & 313 & (18) & 301 & (16) & 357 & (3) \\
\hline 362 & (31) & 370 & (38) & 323 & $(64)$ & 326 & $(60)$ & 367 & $(35)$ \\
\hline 391 & (8) & 434 & (11) & 355 & (15) & 370 & (13) & 400 & (7) \\
\hline 423 & (3) & 446 & (10) & 390 & (19) & 383 & $(31)$ & 437 & (9) \\
\hline 458 & (19) & 502 & (24) & 457 & (41) & 461 & (9) & 464 & (5) \\
\hline 577 & (10) & 536 & $(23)$ & 543 & $(52)$ & 466 & (9) & 550 & $(31)$ \\
\hline 613 & (50) & 603 & (6) & 584 & (16) & 562 & (68) & 625 & (60) \\
\hline 639 & (79) & 650 & (38) & 650 & (27) & 671 & (17) & 633 & (37) \\
\hline 698 & (52) & 678 & (145) & 770 & (9) & 759 & (21) & 688 & (74) \\
\hline 725 & (45) & 729 & (41) & 825 & (2) & 842 & (5) & 733 & (64) \\
\hline 830 & (9) & 838 & (5) & 879 & (27) & 881 & (11) & 859 & (2) \\
\hline 863 & (5) & 865 & (12) & 922 & (50) & 908 & (88) & 880 & (8) \\
\hline 904 & (20) & 930 & (13) & 942 & (15) & 982 & (42) & 900 & (35) \\
\hline 982 & (34) & 981 & (39) & 1020 & (13) & 1005 & (23) & 1025 & (19) \\
\hline 1024 & (34) & 1028 & (12) & 1046 & (98) & 1052 & (16) & 1027 & (67) \\
\hline 1048 & (142) & 1082 & (121) & 1093 & (26) & 1083 & $(42)$ & 1074 & (13) \\
\hline 1084 & (14) & 1104 & (22) & 1094 & (33) & 1094 & (99) & 1103 & (57) \\
\hline 1101 & (8) & 1137 & (76) & 1128 & (29) & 1150 & (14) & 1135 & (136) \\
\hline 1158 & (79) & 1153 & (6) & 1206 & (55) & 1212 & (17) & 1177 & (136) \\
\hline 1177 & (105) & 1178 & (140) & 1266 & (13) & 1262 & (7) & 1197 & (10) \\
\hline
\end{tabular}




\begin{tabular}{cccccccccc}
\hline$\left[\mathrm{N}, \mathrm{CO}, \mathrm{OH}_{\mathrm{s}}\right]$-tggt & \multicolumn{2}{c}{$[\mathrm{N}, \mathrm{CO}]$-tg_g $\mathrm{g}_{+} \mathrm{t}$} & \multicolumn{2}{c}{$\left[\mathrm{CO}_{2}{ }^{-}\right]$-ctgt } & \multicolumn{2}{c}{$\left[\mathrm{CO}_{2}^{-}\right]$-cgtt } & \multicolumn{1}{c}{$[\mathrm{N}, \mathrm{CO}]$-tgtt } \\
\hline $\mathbf{1 2 2 1}$ & $\mathbf{( 5 0 )}$ & $\mathbf{1 2 5 1}$ & $\mathbf{( 6 1 )}$ & $\mathbf{1 3 2 1}$ & $\mathbf{( 4 7 )}$ & $\mathbf{1 3 2 6}$ & $\mathbf{( 5 1 )}$ & $\mathbf{1 2 3 5}$ & $\mathbf{( 2 3 )}$ \\
$\mathbf{1 2 8 0}$ & $\mathbf{( 4 )}$ & $\mathbf{1 2 5 9}$ & $\mathbf{( 1 8 )}$ & $\mathbf{1 3 4 5}$ & $\mathbf{( 5 )}$ & $\mathbf{1 3 4 3}$ & $\mathbf{( 1 3 )}$ & $\mathbf{1 2 8 8}$ & $\mathbf{( 2 )}$ \\
$\mathbf{1 3 2 1}$ & $\mathbf{( 1 8 )}$ & $\mathbf{1 3 4 2}$ & $\mathbf{( 8 )}$ & $\mathbf{1 3 6 2}$ & $\mathbf{( 1 1 )}$ & $\mathbf{1 3 6 9}$ & $\mathbf{( 8 3 )}$ & $\mathbf{1 3 1 9}$ & $\mathbf{( 2 1 )}$ \\
$\mathbf{1 3 4 6}$ & $\mathbf{( 3 )}$ & $\mathbf{1 3 5 1}$ & $\mathbf{( 1 4 )}$ & $\mathbf{1 3 9 1}$ & $\mathbf{( 1 1 )}$ & $\mathbf{1 3 8 9}$ & $\mathbf{( 2 2 )}$ & $\mathbf{1 3 4 1}$ & $\mathbf{( 2 )}$ \\
$\mathbf{1 3 7 1}$ & $\mathbf{( 1 9 )}$ & $\mathbf{1 3 6 9}$ & $\mathbf{( 3 0 )}$ & $\mathbf{1 3 9 9}$ & $\mathbf{( 2 0 6 )}$ & $\mathbf{1 4 0 7}$ & $\mathbf{( 7 8 )}$ & $\mathbf{1 3 6 9}$ & $\mathbf{( 2 5 )}$ \\
$\mathbf{1 3 9 2}$ & $\mathbf{( 6 )}$ & $\mathbf{1 3 9 2}$ & $\mathbf{( 7 )}$ & $\mathbf{1 4 2 7}$ & $\mathbf{( 3 3 6 )}$ & $\mathbf{1 4 1 6}$ & $\mathbf{( 3 6 7 )}$ & $\mathbf{1 3 9 4}$ & $\mathbf{( 2 0 )}$ \\
$\mathbf{1 4 1 4}$ & $\mathbf{( 8 8 )}$ & $\mathbf{1 4 1 3}$ & $\mathbf{( 7 0 )}$ & $\mathbf{1 4 5 5}$ & $\mathbf{( 3 0 )}$ & $\mathbf{1 4 5 7}$ & $\mathbf{( 7 )}$ & $\mathbf{1 4 1 9}$ & $\mathbf{( 6 3 )}$ \\
$\mathbf{1 4 5 2}$ & $\mathbf{( 8 )}$ & $\mathbf{1 4 5 6}$ & $\mathbf{( 4 )}$ & $\mathbf{1 4 6 0}$ & $\mathbf{( 2 5 )}$ & $\mathbf{1 4 6 0}$ & $\mathbf{( 2 3 )}$ & $\mathbf{1 4 5 7}$ & $\mathbf{( 1 5 )}$ \\
$\mathbf{1 4 6 3}$ & $\mathbf{( 9 )}$ & $\mathbf{1 4 6 0}$ & $\mathbf{( 1 2 )}$ & $\mathbf{1 5 6 1}$ & $\mathbf{( 3 6 )}$ & $\mathbf{1 5 9 3}$ & $\mathbf{( 6 3 )}$ & $\mathbf{1 4 5 8}$ & $\mathbf{( 9 )}$ \\
$\mathbf{1 6 1 0}$ & $\mathbf{( 8 1 )}$ & $\mathbf{1 5 9 2}$ & $\mathbf{( 5 6 )}$ & $\mathbf{1 6 2 5}$ & $\mathbf{( 1 5 6 )}$ & $\mathbf{1 6 1 7}$ & $\mathbf{( 3 4 )}$ & $\mathbf{1 5 9 6}$ & $\mathbf{( 8 1 )}$ \\
$\mathbf{1 7 0 5}$ & $\mathbf{( 4 0 2 )}$ & $\mathbf{1 6 8 4}$ & $\mathbf{( 4 8 4 )}$ & $\mathbf{1 6 3 7}$ & $\mathbf{( 6 0 )}$ & $\mathbf{1 6 2 3}$ & $\mathbf{( 1 7 2 )}$ & $\mathbf{1 6 9 0}$ & $\mathbf{( 4 4 6 )}$ \\
2968 & $(2)$ & 2965 & $(4)$ & 2961 & $(6)$ & 2927 & $(18)$ & 2893 & $(34)$ \\
2978 & $(6)$ & 2987 & $(4)$ & 2990 & $(2)$ & 2974 & $(3)$ & 2974 & $(2)$ \\
3011 & $(1)$ & 3001 & $(2)$ & 3027 & $(12)$ & 3036 & $(<1)$ & 3005 & $(1)$ \\
3033 & $(10)$ & 3033 & $(10)$ & 3032 & $(2)$ & 3040 & $(6)$ & 3038 & $(6)$ \\
3040 & $(5)$ & 3034 & $(10)$ & 3037 & $(5)$ & 3056 & $(3)$ & 3055 & $(5)$ \\
3388 & $(38)$ & 3324 & $\mathbf{( 7 0 )}$ & 3140 & $(188)$ & 3188 & $(39)$ & 3315 & $(118)$ \\
3450 & $(48)$ & 3444 & $(61)$ & 3229 & $(177)$ & 3238 & $(307)$ & 3409 & $(67)$ \\
3586 & $(189)$ & 3591 & $(193)$ & 3414 & $(109)$ & 3396 & $(110)$ & 3583 & $(186)$ \\
3697 & $(116)$ & 3704 & $(97)$ & 3708 & $(105)$ & 3703 & $(113)$ & 3702 & $(102)$
\end{tabular}

${ }^{a}$ Bold indicates the region of the IR spectrum included in the present experimental study. 
Table S8. Bond Distances $(\AA)$ and Bond Angles (deg) for $\mathrm{CdCl}^{+}(\mathrm{Thr})$ Structures $^{a}$

\begin{tabular}{|c|c|c|c|c|c|c|}
\hline structure & $\mathrm{r}(\mathrm{M}-\mathrm{X})$ & $\mathrm{r}(\mathrm{M}-\mathrm{O})$ & $\mathrm{r}\left(\mathrm{M}-\mathrm{O}_{\mathrm{s}}\right)$ & $\angle \mathrm{XMO}$ & $\angle \mathrm{XMO}_{\mathrm{s}}$ & $\angle \mathrm{OMO}_{\mathrm{s}}$ \\
\hline$\left[\mathrm{N}, \mathrm{CO}, \mathrm{OH}_{\mathrm{s}}\right]-\operatorname{tggt}$ & 2.32 & 2.39 & 2.48 & 70.3 & 69.3 & 76.8 \\
\hline$[\mathrm{N}, \mathrm{CO}]-\operatorname{tg} \mathrm{g}_{+}+\mathrm{t}$ & 2.29 & 2.29 & & 73.6 & & \\
\hline$\left[\mathrm{CO}_{2}^{-}\right]$-ctgt & $2.65^{b}$ & 2.13 & & $54.2^{b}$ & & \\
\hline$\left[\mathrm{CO}_{2}^{-}\right]-\mathrm{cgtt}$ & $2.65^{b}$ & 2.14 & & $54.1^{b}$ & & \\
\hline$[\mathrm{N}, \mathrm{CO}]$-tgtt & 2.26 & 2.34 & & 72.8 & & \\
\hline$[\mathrm{N}, \mathrm{CO}]-\operatorname{tgtg}$ & 2.26 & 2.36 & & 72.8 & & \\
\hline$\left[\mathrm{N}, \mathrm{CO}, \mathrm{OH}_{\mathrm{s}}\right]-\mathrm{cggt}$ & 2.36 & 2.33 & 2.49 & 70.5 & 68.3 & 76.9 \\
\hline$\left[\mathrm{N}, \mathrm{OH}_{\mathrm{s}}\right]-\mathrm{tg}_{-} \mathrm{tt}$ & 2.23 & & 2.35 & & 73.0 & \\
\hline$\left[\mathrm{N}, \mathrm{OH}_{\mathrm{s}}, \mathrm{OH}\right]-$ tggt & 2.26 & $2.64^{c}$ & 2.40 & $64.3^{c}$ & 72.1 & $76.4^{c}$ \\
\hline$[\mathrm{N}, \mathrm{CO}]-\mathrm{ccgt}$ & 2.40 & 2.20 & & 73.2 & & \\
\hline$\left[\mathrm{N}, \mathrm{OH}_{\mathrm{s}}\right]-\mathrm{tg}_{+} \mathrm{tt}$ & 2.24 & & 2.34 & & 72.8 & \\
\hline$[\mathrm{N}, \mathrm{CO}]-\operatorname{tg}_{+} \mathrm{g}_{-} \mathrm{t}$ & 2.32 & 2.27 & & 73.3 & & \\
\hline$\left[\mathrm{CO}, \mathrm{OH}_{\mathrm{s}}\right]-\mathrm{cggt}$ & & 2.13 & 2.39 & & & 80.5 \\
\hline$[\mathrm{N}, \mathrm{CO}]$-tggg & 2.33 & 2.27 & & 73.3 & & \\
\hline$[\mathrm{N}, \mathrm{CO}]-\mathrm{cgtt}$ & 2.29 & 2.31 & & 72.9 & & \\
\hline$\left[\mathrm{CO}_{2}^{-}\right]-\operatorname{cggg}$ & $2.68^{b}$ & 2.13 & & $53.6^{b}$ & & \\
\hline$\left[\mathrm{CO}_{2}^{-}\right]$-cggt & $2.81^{b}$ & 2.10 & & $51.6^{b}$ & & \\
\hline$[\mathrm{N}, \mathrm{OH}]$-tgtt & 2.20 & $2.53^{c}$ & & $69.5^{c}$ & & \\
\hline
\end{tabular}

${ }^{a}$ Values calculated at the B3LYP/def2-TZVP level of theory. Except as noted, $\mathrm{X}=$ amino nitrogen, $\mathrm{O}=$ carbonyl oxygen of backbone, and $\mathrm{O}_{\mathrm{s}}=$ side-chain hydroxyl oxygen. ${ }^{b} \mathrm{X}=$ second oxygen in carboxylate group. ${ }^{c} \mathrm{O}=$ oxygen of backbone hydroxyl group. 


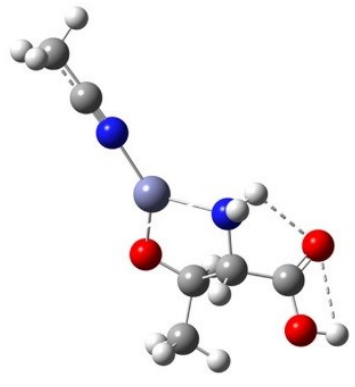

$\left[\mathrm{N}, \mathrm{O}_{\mathrm{s}}^{-}\right]-\operatorname{tg} \_\mathrm{t}(\mathrm{ACN})$

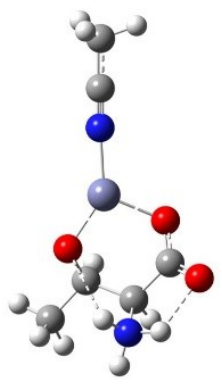

$\left[\mathrm{CO}^{-}, \mathrm{O}_{\mathrm{s}}^{-}\right]\left(\mathrm{NH}_{3}{ }^{+}\right)-\mathrm{cg}(\mathrm{ACN})$

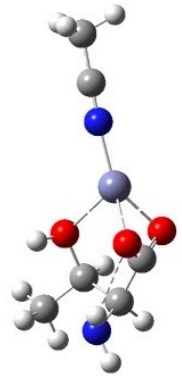

$\left[\mathrm{CO}_{2}{ }^{-}, \mathrm{OH}_{\mathrm{s}}\right]-\mathrm{gcg}(\mathrm{ACN})$

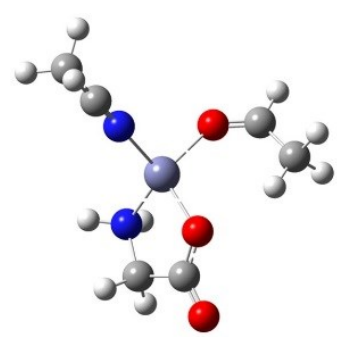

$\mathrm{Zn}(\mathrm{Gly}-\mathrm{H})^{+}\left[\mathrm{N}, \mathrm{CO}^{-}\right]$

$\left(\mathrm{CH}_{3} \mathrm{CHO}\right)(\mathrm{ACN})$

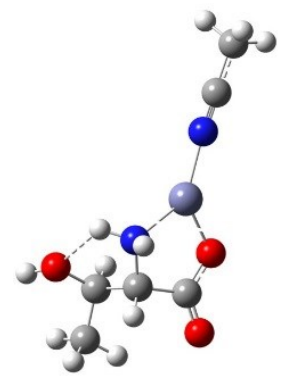

$\left[\mathrm{N}, \mathrm{CO}^{-}\right]-\mathrm{gtt}(\mathrm{ACN})$

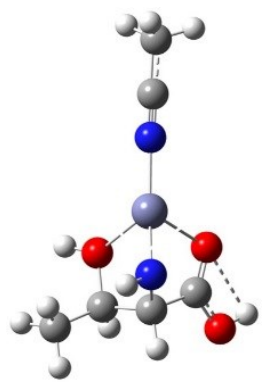

$\left[\mathrm{N}^{-}, \mathrm{CO}, \mathrm{OH}_{\mathrm{s}}\right]-\operatorname{tggt}(\mathrm{ACN})$

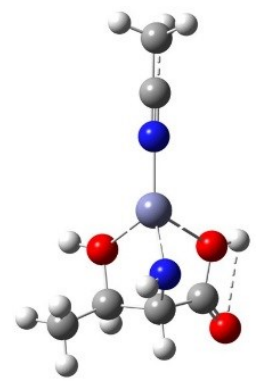

$\left[\mathrm{N}^{-}, \mathrm{OH}_{\mathrm{s}}, \mathrm{OH}\right]-\operatorname{tggt}(\mathrm{ACN})$

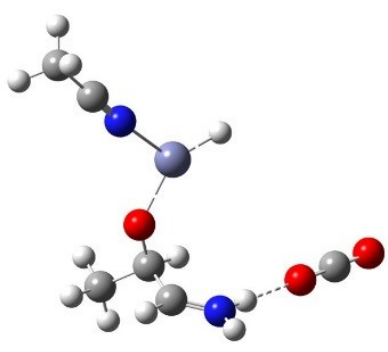

$\mathrm{ZnH}\left(\mathrm{C}_{3} \mathrm{H}_{7} \mathrm{ON}\right)^{+}\left(\mathrm{CO}_{2}\right)(\mathrm{ACN})$

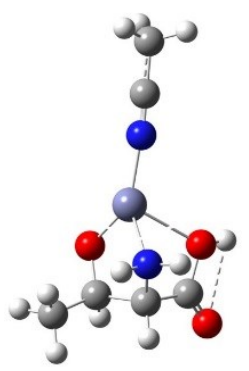

$\left[\mathrm{N}, \mathrm{O}_{\mathrm{s}}{ }^{-}, \mathrm{OH}\right]-\operatorname{tgg}(\mathrm{ACN})$

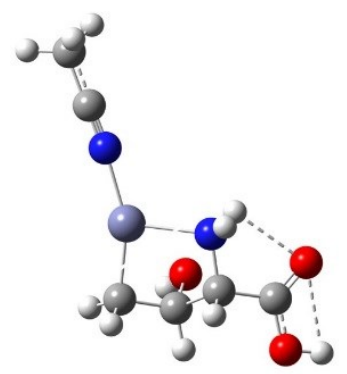

$\left[\mathrm{N}, \mathrm{C}_{\mathrm{Y}}{ }^{-}\right]-\mathrm{tg} \_\mathrm{g} \_\mathrm{t}(\mathrm{ACN})$
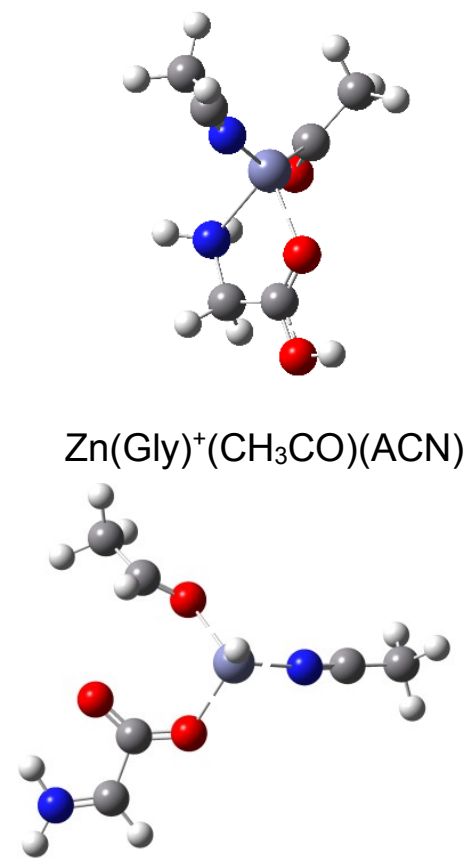

$\mathrm{ZnH}(\mathrm{Gly}-2 \mathrm{H})^{+}\left(\mathrm{CH}_{3} \mathrm{CHO}\right)(\mathrm{ACN})$

Figure S1 


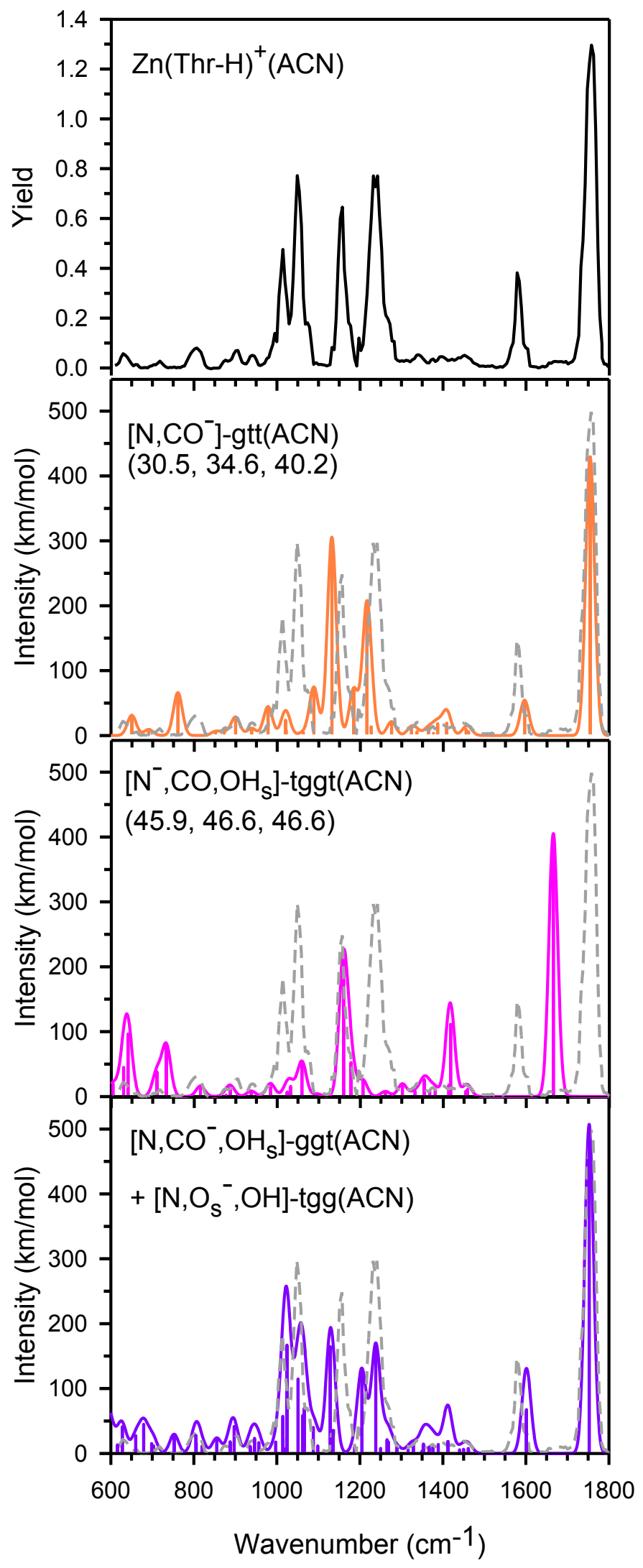

Figure S2 


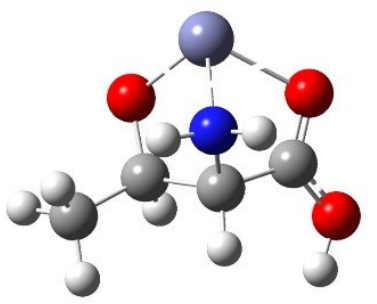

$\left[\mathrm{N}, \mathrm{Os}^{-}, \mathrm{CO}\right]-\mathrm{cgg}$

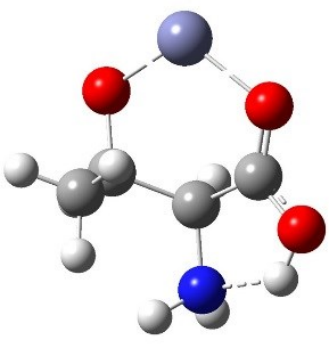

$\left[\mathrm{O}^{-}, \mathrm{CO}\right]-\mathrm{cgg}$

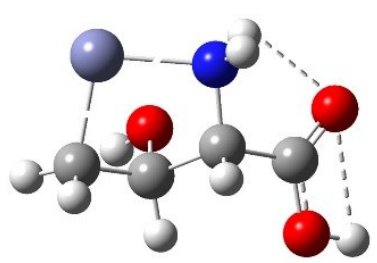

$\left[\mathrm{N}, \mathrm{C}_{\mathrm{Y}}{ }^{-}\right]-\mathrm{tg}{ }_{-} \mathrm{g}$. $\mathrm{t}$

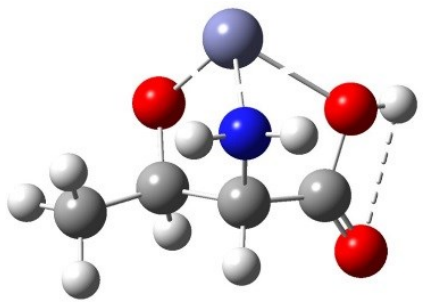

$\left[\mathrm{N}, \mathrm{Os}^{-}, \mathrm{OH}\right]-\operatorname{tgg}$

Figure S3 


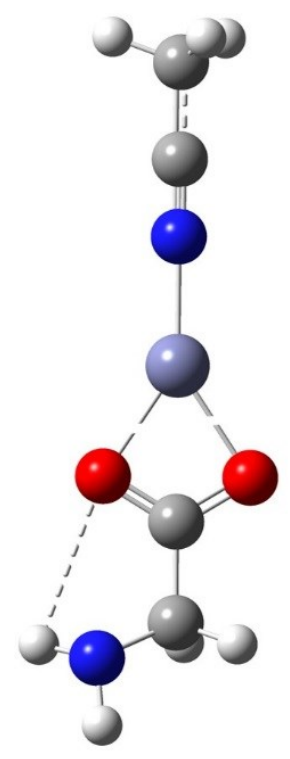

$\mathrm{Zn}(\mathrm{Gly}-\mathrm{H})^{+}\left[\mathrm{CO}_{2}^{-}\right]-\mathrm{C}, \mathrm{NH} \cdot \mathrm{CO}(\mathrm{ACN}) \quad \mathrm{Zn}(\mathrm{Gly}-\mathrm{H})^{+}[\mathrm{N}, \mathrm{CO}]\left(\mathrm{C}_{\alpha^{-}}\right)^{-\mathrm{tt}}(\mathrm{ACN})$

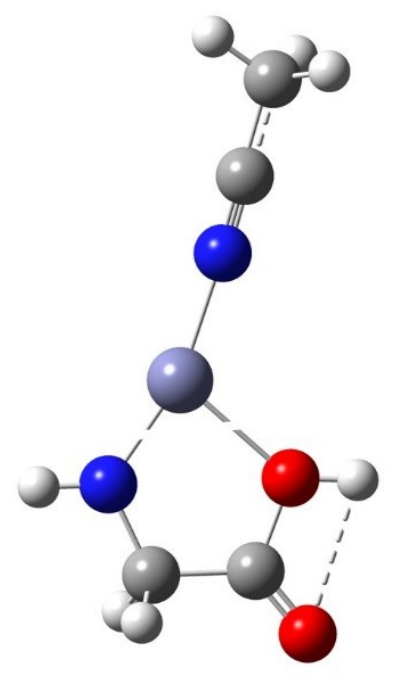

$\mathrm{Zn}(\mathrm{Gly}-\mathrm{H})^{+}\left[\mathrm{N}^{-}, \mathrm{OH}\right]-\mathrm{tc}(\mathrm{ACN})$
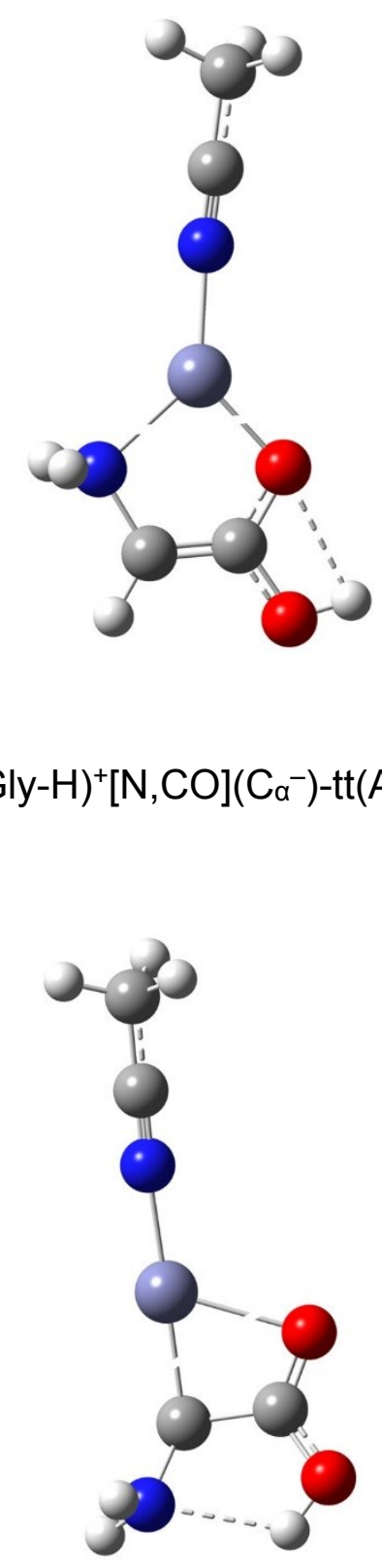

$\mathrm{Zn}(\mathrm{Gly}-\mathrm{H})^{+}\left[\mathrm{C}_{\alpha^{-}}, \mathrm{CO}\right]-\mathrm{cc}(\mathrm{ACN})$

Figure S4 


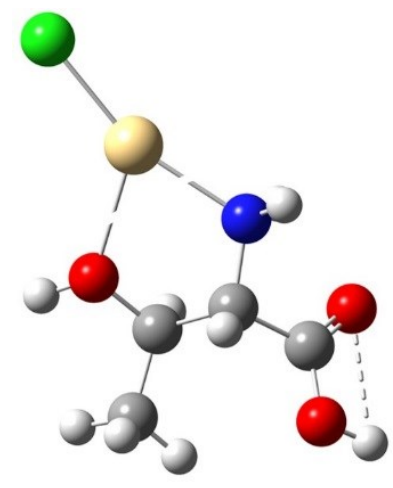

$\left[\mathrm{N}, \mathrm{OH}_{\mathrm{s}}\right]-\mathrm{tg} \mathrm{tt}_{\mathrm{t}}$

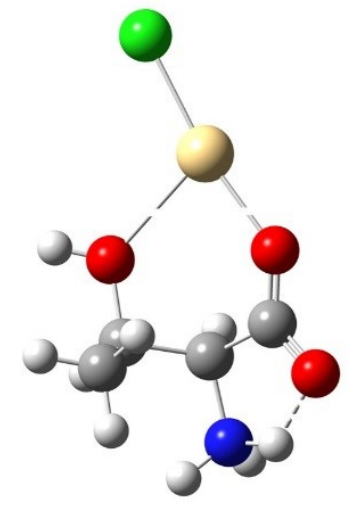

$\left[\mathrm{CO}^{-}, \mathrm{OH}_{\mathrm{s}}\right]-\mathrm{ggt}$

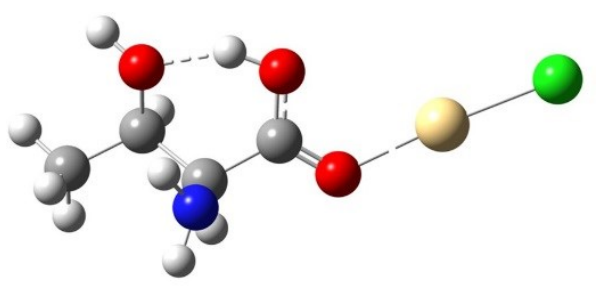

[CO]-ccg+t

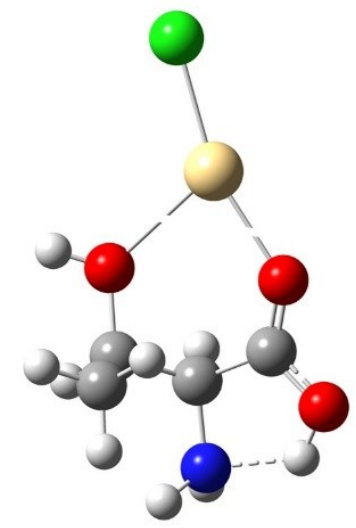

$\left[\mathrm{CO}, \mathrm{OH}_{\mathrm{s}}\right]-\mathrm{cggt}$
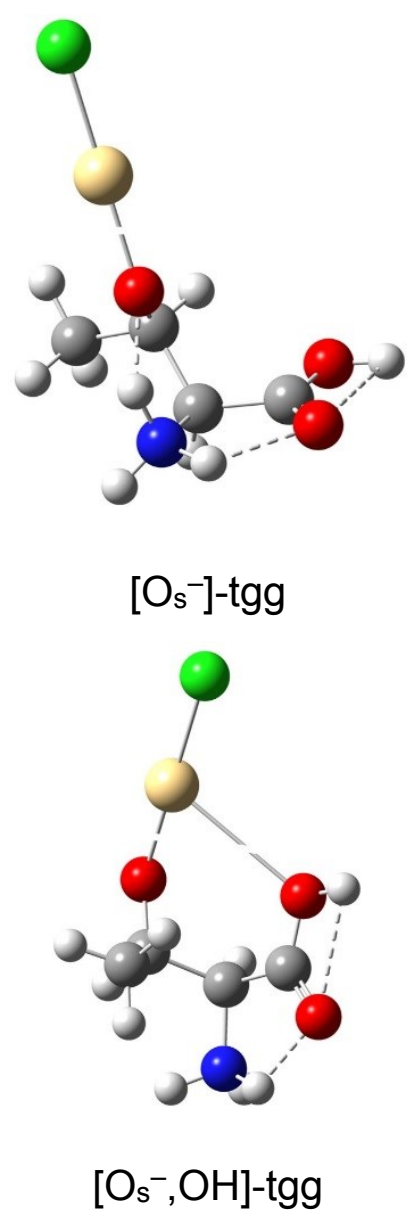

Figure S5 